Sharif University of Technology
Scientia Iranica
SCIENTIA

\title{
Computational investigation into vortex breakdown over a pitching delta wing at supersonic speeds
}

\author{
M. Hadidoolabi and H. Ansarian* \\ Department of Aerospace Engineering, Malek Ashtar University of Technology, Lavizan, Tehran, Iran.
}

Received 14 February 2016; received in revised form 10 October 2016; accepted 29 October 2016

\section{KEYWORDS}

Delta wing;

Pitching oscillation;

Vortex breakdown;

Supersonic flow;

Aerodynamic

coefficients

\begin{abstract}
Vortex breakdown in compressible flows over a 60-degree sweep delta wing with a sharp leading edge undergoing pitching oscillations is computationally studied. Emphasis in this study is on possible supersonic vortex breakdown for pitching motion of a delta wing, as well as behavior of the aerodynamic characteristics during a cycle. Unstructured grid, $k-\omega$ SST turbulence model, and dual-time implicit time integration are used. Accurate simulations are performed for various Mach numbers and mean angles of attack to cover different flow structures and phenomena associated with them. Variations of flow structure around the wing and hysteresis loops associated with lift coefficient and vortex breakdown location during a pitching cycle are investigated. The trends with Mach number, mean angle of attack, amplitude of pitching, and pitching frequency are illustrated.

(C) 2017 Sharif University of Technology. All rights reserved.
\end{abstract}

\section{Introduction}

Delta wings are used in many aircrafts, which fly at supersonic speeds. Airplanes and space planes with delta wing often fly at high angles of attack, especially in take-off or landing phases, where their aerodynamic performance at low speeds is weak. Moreover, flying at high angles of attack even at transonic and supersonic regimes can be expected from future space planes in the reentry phase. As the maneuverability of such aircraft enhances, the comprehension of the nature of unsteady flows around delta wings becomes more important. In particular, if computational models are to be developed for time-dependent motions of the aircraft or its aerodynamic surfaces, it is necessary to study the features such as vortex breakdown and vortex-shock patterns on delta wings and the associated physical mechanisms.

*. Corresponding author.

E-mail addresses: mhadidoolabi@mut.ac.ir (M. Hadidoolabi); ansarianh@mut.ac.ir (H. Ansarian)

doi: $10.24200 /$ sci. 2017.4246
It is well known that in the steady flight of a delta wing, a shear layer is separated from the leading edge, which produces two counter-rotating vortices on the leeside of the wing. This results in production of large suction peaks and, thereby, generation of lift. Two much smaller vortices with opposite sense of rotation relative to primary vortices, i.e. the secondary vortices, are also formed in certain flow conditions. At sufficiently high angles of attack, the leading edge vortices above a delta wing undergo a vigorous form of flow discontinuity known as vortex breakdown. This phenomenon is characterized by vortex core inflation, internal axial flow stagnation, and substantial fluctuations in flow variables downstream.

The sudden onset of vortex breakdown as well as the consequent lags and hysteresis, which appear in aerodynamic loads, can have severe impacts on stability and control of high-performance aircraft and may lead to the reduction in its operational envelope. Moreover, the coherent fluctuations characteristic of vortex breakdown may assist undesirable fluid/structure interactions among aircraft components intersecting the vortex path. 
Vortex breakdown on stationary delta wings at incompressible regime has been the focus of many efforts. Gursul et al. [1,2] presented extensive reviews of the steady and unsteady aerodynamics of delta wings flying at low speeds.

The unsteady flow structure on a delta wing undergoing maneuver has received less attention. Such research is motivated by the need to enhance the maneuver capabilities of current and future combat airplanes, missiles, and spacecraft. When a delta wing is subjected to pitching, plunging, or any other type of unsteady motion, a time lag is seen in the response of the vortex flow. This usually results in a temporary delay in vortex formation at low angles of attack or temporary delay in vortex breakdown at higher angles of attack. For delta wings subjected to periodic motions, a hysteresis develops in the flow characteristics relative to the stationary wing, which may increase with motion frequency. Using these unsteady effects, a high-performance aircraft can be able to perform certain maneuvers more quickly and efficiently.

Rockwell [3] and Visbal [4] reviewed the unsteady aspects of flow structures and vortex breakdown on delta wings. For sinusoidal oscillation of a delta wing with highly reduced frequency, Atta and Rockwell [5] observed the existence of vortex core over only a fraction of the motion cycle, and occurrence of the maximum vortex breakdown position near the maximum angle of attack, instead of at the minimum angle of attack, which is expected from the quasi-steady assumptions. LeMay et al. [6] studied the response of breakdown position on a delta wing to a harmonic pitching motion and the effect of reduced frequency. By performing unsteady pressure measurements on a pitching delta wing, Gursul and Yang [7], based on the suggestion of Gursul and Ho [8] that vortex breakdown over delta wings in unsteady flow was caused by the external pressure gradient, showed that the phase lag of the vortex breakdown position on the wing might be related to the adverse pressure gradient and its variations on the wing surface. Lin and Rockwell [9] defined the region of vortex breakdown in terms of patterns of azimuthal vorticity, as well as critical points of the sectional topology. Jones et al. [10] studied the relation between the appearance of negative azimuthal vorticity and the onset of vortex breakdown over a delta wing using computational simulations of flow over the static and pitching delta wing. Goruney and Rockwell [11] investigated the near-surface flow structure and topology on a delta wing of moderate sweep angle using a technique of high-image-density digital particle image velocimetry. They studied the time evolution of the surface topology during relaxation of the flow after termination of a pitching maneuver for a wide range of pitch rates. Jian et al [12] used and
Improved Delayed Detached Eddy Simulation (IDDES) method based on the $k-\omega \mathrm{SST}$ turbulence model to predict the unsteady vortex breakdown past an $80^{\circ} / 65^{\circ}$ double-delta wing. They analyzed the fluctuations of mean lift, drag, pitching moment, pressure coefficients, and breakdown locations.

Most studies of unsteady vortical flow structure on oscillating delta wings have been done for incompressible flows or for flows with very low free-stream Mach number. As a result, little is known about the onset and transient behavior of breakdown on a pitching delta wing at higher Mach numbers, especially at supersonic speeds. When flying with much higher speeds, flow becomes more complicated since shock waves appear in the flow, which interact with vortices. At supersonic velocities, there may be various flow regimes on the leeward side of delta wings, which differ by the numbers and positions of streamwise vortices, internal shock waves, and other features of the flow. The earliest effort to understand supersonic flow pattern over delta wings for various wing geometries and various flow conditions appeared in the work by Stanbrook and Squire [13]. They examined all the experimental data available and proposed a classification for the flow patterns based on the component of Mach number normal to the leading edge, $M_{N}$, and the component of angle of attack normal to the leading edge, $\alpha_{N}$. They introduced two types of flows, namely, attached flow and separated flow, at the leading edge. These two types were separated by a boundary line near $M_{N}=1$, which was called the Stanbrook-Squire boundary (Figure 1). Parameters $\alpha_{N}$ and $M_{N}$ were calculated as follows:

$$
\begin{aligned}
& \alpha_{N}=\tan ^{-1}(\tan \alpha / \cos \Lambda), \\
& M_{N}=M_{\infty} \cos \Lambda \sqrt{1+\sin ^{2} \alpha \tan ^{2} \Lambda} .
\end{aligned}
$$

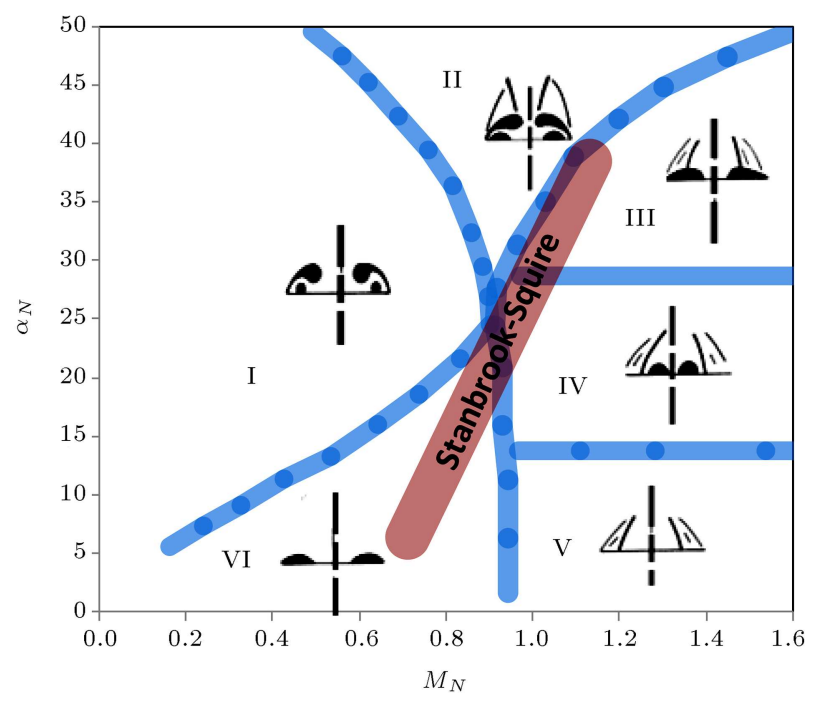

Figure 1. Flow classification. 
Miller and Wood [14] experimentally investigated the flow patterns over delta wings with various sweep angles using several visualization methods. They introduced six types of flow patterns based on $\alpha_{N}$ and $M_{N}$ :

(I) Classical vortex;

(II) Vortex with shock;

(III) Separation bubble with shock;

(IV) Shock-induced separation;

(V) Shock with no separation;

(VI) Separation bubble with no shock.

These patterns are shown in Figure 1. Szodruch and Peake [15] proposed a similar classification for delta wings with more thickness than those studied by Miller and Wood. Seshadri and Narayan [16], Brodetsky et al. [17], and Brodetsky and Shevchenko [18] proposed similar classifications by examining flow fields in more detail. Imai et al. [19] conducted computational simulations of flow field over a $65^{\circ}$ sweep delta wing and investigated the flow mechanism behind the flow classification at high angles of attack in transonic and supersonic regimes. Oyama et al. [20] performed wind tunnel experiments to examine the effect of Mach number on flow structure over a delta wing with blunt leading edges in supersonic and high angle of attack regions.

Literature review shows that there has not been significant research, either computational or experimental, performed for steady or unsteady aspects of supersonic vortex breakdown over delta wings. In this study, flow fields over a $60^{\circ}$ pitching delta wing with sharp leading edge at various mean angles of attack, from subsonic to supersonic regimes, are computationally simulated and the results are analyzed. The objective of the present study is to investigate when the vortex breakdown occurs in supersonic flow and how its location along the wing chord varies during a pitching cycle. Also, the corresponding aerodynamic characteristics of hysteresis loops are studied. The effects of frequency, Mach number, pitching amplitude, and mean angle of attack are investigated.

\section{Methodologies}

\subsection{Computational methods}

Three-dimensional unsteady compressible NavierStokes equations are solved as the governing equations using a valid CFD code. Numerical fluxes for the convective terms are computed using the Roe scheme, and the upwind MUSCL algorithm is applied to extend the spatial accuracy to 2 nd order based on the primitive variables. The viscous fluxes are computed using the 2nd order central differencing. The flow field is assumed to be fully turbulent, and the two-equation $k-\omega \mathrm{SST}$ turbulence model is applied. Different versions of $k-\omega$ turbulent model have been found to be appropriate for capturing vortex flow over delta wings by several researchers [21-24]. Schiavetta et al. [22] compared the Detached Eddy Simulation (DES) and Unsteady Reynolds-Averaged Navier-Stokes (URANS) turbulence modeling methods for prediction of unsteady vortical flows over delta wings. They concluded that URANS turbulence models were able to predict the dominant features of the low-frequency phenomenon present in the vortex system. An implicit dual-time algorithm was applied for the unsteady time integration. Density was calculated by ideal gas law and viscosity by Sutherland's law.

\subsection{Model geometry and grid}

The model geometry is illustrated in Figure 2. As shown in the figure, the analyzed delta wing has leading-edge sweep angle of $60^{\circ}$. The upper surface is flat and the leading edge is sharp $\left(10^{\circ}\right.$ angle normal to leading edge located on lower surface) to minimize the effect of leading edge shape on the flow field. Wing thickness ratio is 0.03 based on the root chord length. The model is geometrically similar to that of Miller and Wood [14]. There is no sideslip (yaw) angle, thus the flow field must be considered to be symmetric on the center line of the wing. Therefore, only half of the wing is covered by the computational domain. This approach neglects the potential flow asymmetry over the wing, but has been found to exhibit enough accuracy for low to moderate angles of attack [10,22,23,25]. The simulations were performed on an unstructured grid (Figure 3 ), which approximately had $7.0 \times 10^{6}$ cells and was obtained after excessive simulation for grid independence study.

Since the objective of this study was to simulate the flow structure accurately, grid study was performed by checking the convergence of the numeric values of flow field variables in several flow field points for several steady flow conditions. An example is shown in Table 1

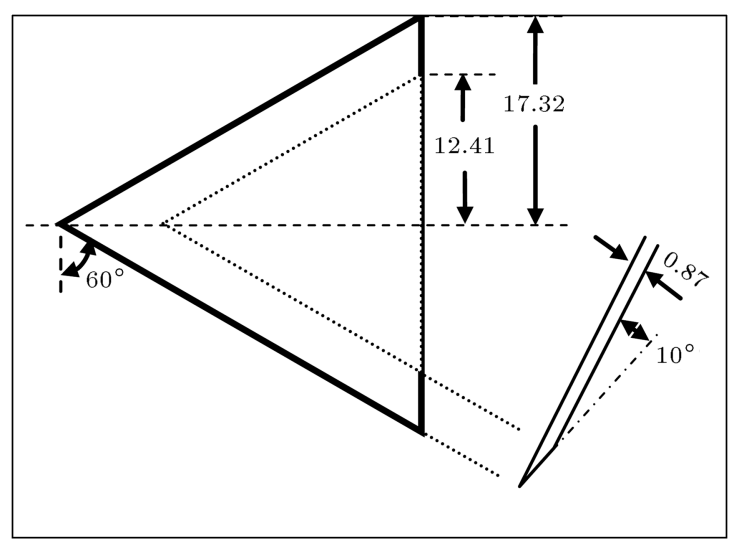

Figure 2. Model geometry. 


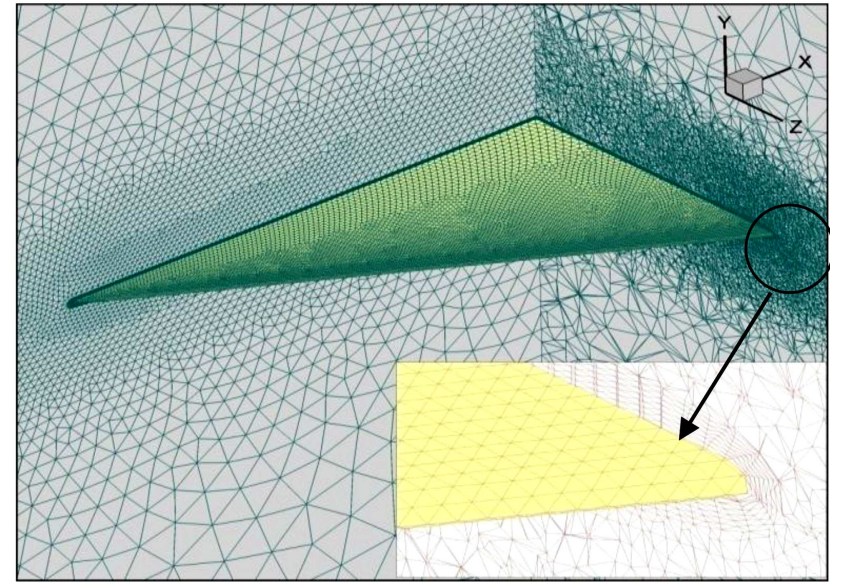

Figure 3. Computational grid.

Table 1. Axial and tangential velocity components in an arbitrary point for various grid sizes.

\begin{tabular}{cccc}
\hline Grid size $\times \mathbf{1 0}^{\mathbf{6}}$ & 5.1 & 7.0 & 9.2 \\
$\boldsymbol{u} / \boldsymbol{U}_{\infty}$ & 1.2511 & 1.2461 & 1.2460 \\
$\boldsymbol{v} / \boldsymbol{U}_{\infty}$ & 0.4901 & 0.4830 & 0.4828 \\
\hline
\end{tabular}

for the average non-dimensional axial and tangential velocities in a selected point near the vortex core. This point was located in a crossflow plane at $30 \%$ chordwise station, for the flow with Mach number of 1.2 and angle of attack of $20^{\circ}$.

The grid had a first wall spacing of $1<y^{+}<6$, which was appropriate for the turbulent model used. The grid was refined within the vortex core region to improve the grid quality for capturing the vortices well. The upper and lower surfaces of the wing were specified as solid wall with no-slip boundary conditions. Symmetry boundary condition was applied for the symmetry plane. The remaining domain was a hemisphere with diagonal of 15 root chord length and was specified as pressure farfield. The converged steady-state solution was used as the initial condition.

\subsection{Flow conditions}

To examine the possible breakdown behaviors in different supersonic flow structures over the delta wing, the flow conditions in this study were chosen to cover the classification chart of Miller and Wood [14]. The flow and pitch oscillation conditions numerically simulated in the present work are shown in Table 2. Figure 4 shows the flow conditions plotted over the classification chart of Miller and Wood. The large symbols in the figure denote the mean state and the small symbols denote the angle of attack extremes. Small and large arrows represent the oscillations with amplitudes of $4^{\circ}$ and $8^{\circ}$, respectively. Free-stream Mach numbers are $0.4,0.8,1.2$, and 2.0. For the subsonic Mach numbers, only one condition (mean angle of $20^{\circ}$, amplitude of $4^{\circ}$, and frequency of $10 \mathrm{~Hz}$ ) is selected. For the
Table 2. Flow conditions.

\begin{tabular}{ccccc}
\hline $\boldsymbol{M}_{\boldsymbol{\infty}}$ & $\begin{array}{c}\boldsymbol{\alpha}_{\mathbf{0}} \\
(\mathbf{d e g})\end{array}$ & $\begin{array}{c}\overline{\boldsymbol{\alpha}} \\
(\mathbf{d e g})\end{array}$ & $\begin{array}{c}\boldsymbol{f} \\
(\mathbf{H z})\end{array}$ & $\boldsymbol{k}$ \\
\hline 0.4 & 20 & 4 & 10 & 0.076 \\
0.8 & 20 & 4 & 10 & 0.04 \\
1.2 & 8 & 4,8 & $5,10,50$ & $0.013,0.027,0.133$ \\
1.2 & 20 & 4,8 & $5,10,50$ & $0.013,0.027,0.133$ \\
1.2 & 30 & 4,8 & $5,10,50$ & $0.013,0.027,0.133$ \\
2 & 8 & 4,8 & $5,10,50$ & $0.008,0.016,0.08$ \\
2 & 20 & 4,8 & $5,10,50$ & $0.008,0.016,0.08$ \\
2 & 30 & 4,8 & $5,10,50$ & $0.008,0.016,0.08$ \\
2 & 40 & 4 & 10 & 0.016 \\
\hline
\end{tabular}

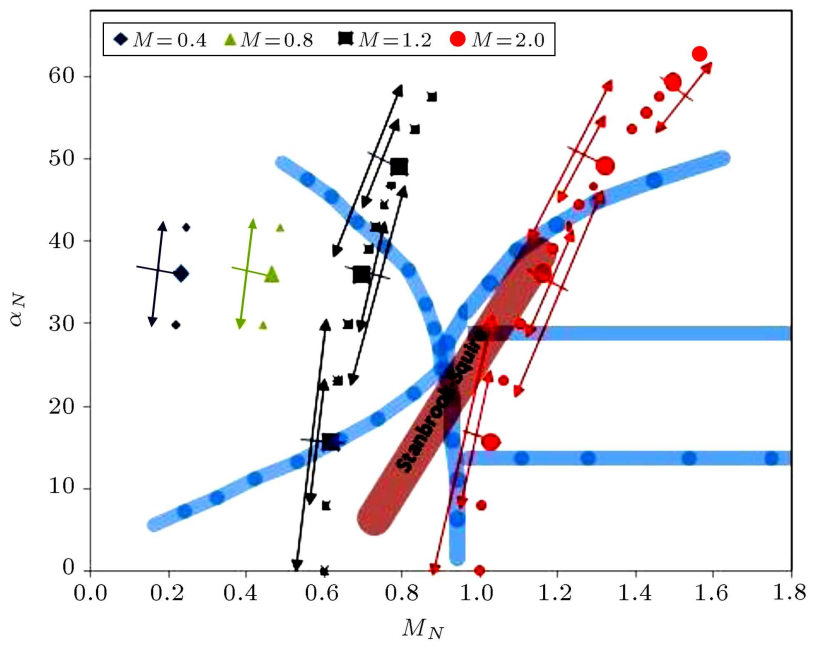

Figure 4. Flow conditions.

supersonic Mach numbers, three mean angles of attack, two amplitudes, and three frequencies of motion, plus an additional condition for $M_{\infty}=2$, are selected to study the effect of each parameter in supersonic conditions. For each condition, the Reynolds number based on the wing root-chord length is $1.3 \times 10^{6}$, similar to the experiments of Miller and Wood [14]. Continuous sinusoidal pitching is performed and the pitching axis is located half maximum thickness below the half root chord location. The wing motion is described as follows:

$$
\alpha=\alpha_{0}+\bar{\alpha} \sin (\omega t+\pi) .
$$

It is important to note that because of large magnitudes of free-stream speed in supersonic regime, reduced frequency, $k=\pi f c / U_{\infty}$, has not a large value even at high frequencies.

\section{Results and discussions}

An extensive post-processing effort was made to understand the physical phenomena occurring in the flow. 
Among the 39 cases mentioned above, some conditions in which vortex breakdown occurs are presented here.

\subsection{Flow patterns}

Before we investigate the vortex breakdown phenomena over the pitching delta wing, it is useful to show the numerical simulation results of some flow patterns introduced by classification chart of Miller and Wood [14]. Details of these vortical flow structures and how they change during a pitching motion are not the subject of this paper. Instead, the breakdown behaviors associated with them are discussed in the next section. The authors [24] have simulated the various crossflow patterns over the stationary and pitching delta wing and have verified their results with experimental data.

Figure 5 shows local Mach number contour map and total pressure contour lines in the crossflow plane at $30 \%$ chordwise station for the flow with free-stream Mach number of 0.8 and angle of attack of $20^{\circ}$. This type of flow is classified into "classical vortex (type (I) )" characterized by the primary and secondary vortices with no shock waves. However, a crossflow shock wave arises below the primary vortex core. Adverse pressure gradient due to this shock wave enhances the secondary flow separation. Flow with Mach number of 1.2 and angle of attack of $20^{\circ}$ would have a similar pattern as it is indicated in the chart. Figure 6 shows the similar map for flow with Mach number of 1.2 and angle of attack of $30^{\circ}$. The structure of flow with these conditions is "vortex with shock (type (II))". The spanwise flow acceleration induced by the primary vortex leads to the appearance of the shock wave over the primary vortex, which is a weak oblique shock in this condition. This shock decelerates flow toward the wing root and changes the flow direction. In Figure 7, the crossflow pattern for the flow with Mach number of 2 and angle of attack of $40^{\circ}$ is shown. The "vortex with shock" pattern in this condition is

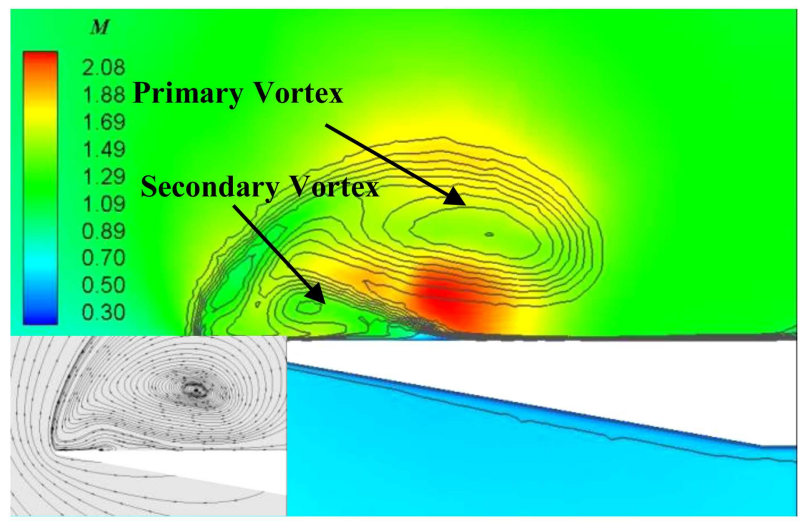

Figure 5. Local Mach number contour map and total pressure contour lines in the crossflow plane at $30 \%$ chordwise station for flow with $M_{\infty}=0.8$, and $\alpha=20^{\circ}$.

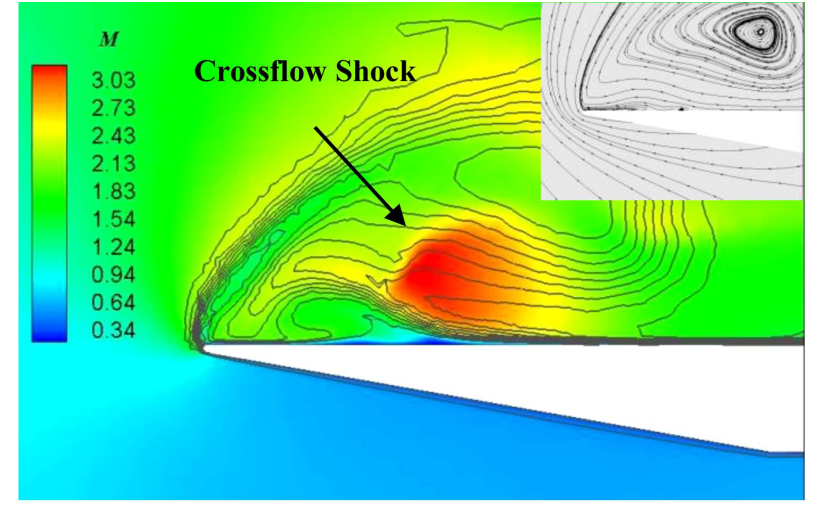

Figure 6. Local Mach number contour map and total pressure contour lines in the crossflow plane at $30 \%$ chordwise station for flow with $M_{\infty}=1.2$, and $\alpha=30^{\circ}$.

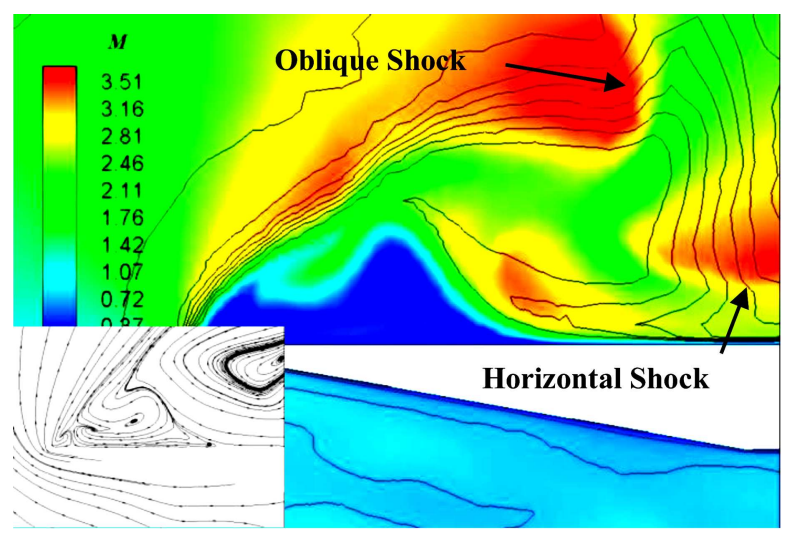

Figure 7. Local Mach number contour map and total pressure contour lines in the crossflow plane at $30 \%$ chordwise station for flow with $M_{\infty}=2.0$, and $\alpha=40^{\circ}$.

accompanied by a horizontal shock wave between the pair of primary vortices. Also, it can be seen in the figure that secondary separation has experienced the breakdown although the primary vortex still exists.

\subsection{Vortex breakdown}

Figures 8 to 11 show the static pressure coefficient and Mach number contours over the leeward side of the wing and the flow field around it for a pitching cycle at four flow conditions. At the left-hand side of these figures are shown the pressure coefficient distribution in three crossflow planes at $30 \%, 60 \%$, and $90 \%$ chordwise stations and a plane crossing the primary vortex axis (upper picture), and the pressure coefficient distribution at the wing surface and the symmetry plane (lower picture). At the right side of these figures are shown the local Mach number distribution in a plane crossing the primary vortex axis, and the crossflow plane at $60 \%$ chordwise station (upper picture) and $90 \%$ chordwise station (lower picture). The figures show one oscillation cycle of the wing by $T / 8$ time steps. Instantaneous time and angle of attack are shown below each snapshot. All unsteady results presented in this paper are extracted 


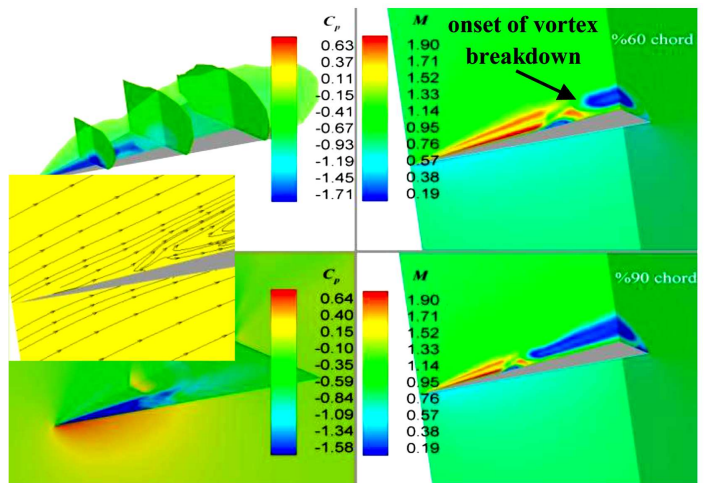

(a) $t=0, \alpha=20^{\circ}$

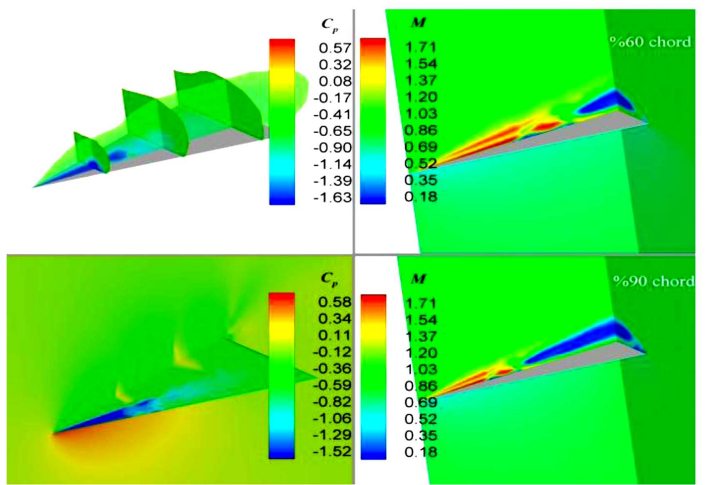

(b) $t=T / 8, \alpha=17.17^{\circ}$

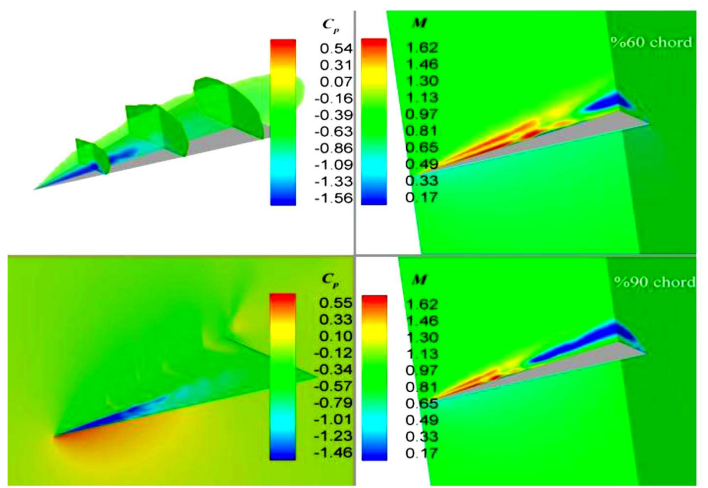

(c) $t=T / 4, \alpha=16^{\circ}$

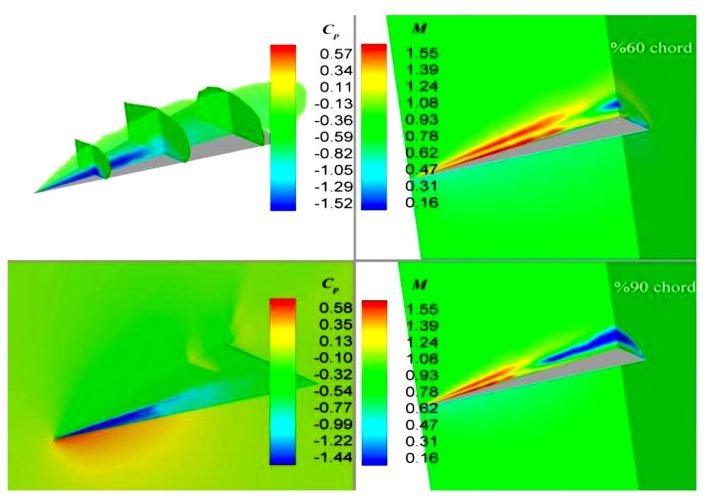

(d) $t=3 T / 4, \alpha=17.17^{\circ}$

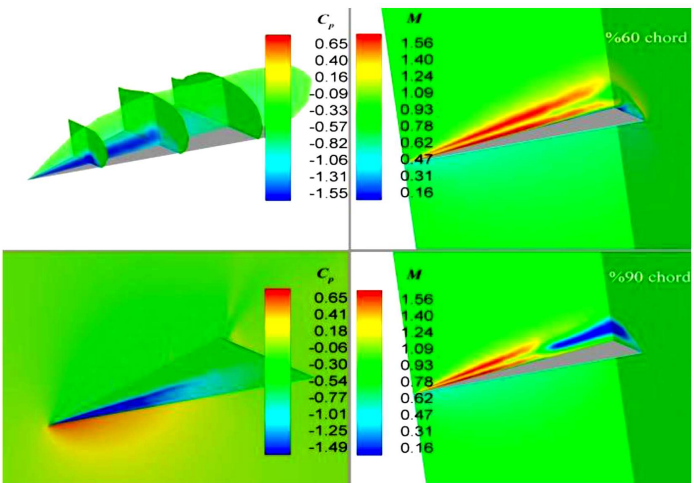

(e) $t=T / 2, \alpha=20^{\circ}$

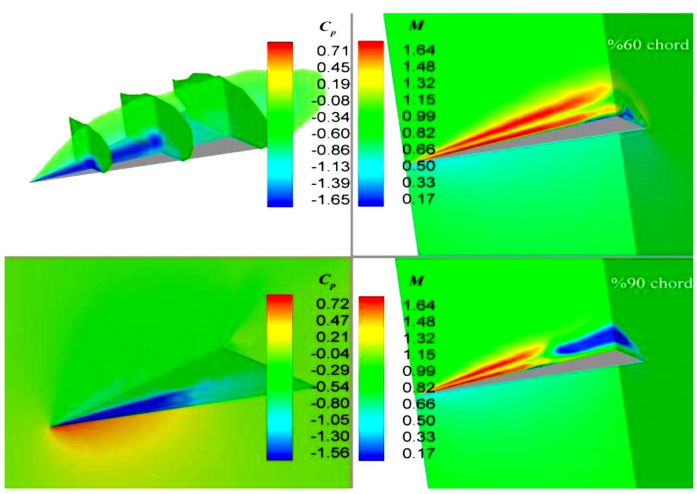

(f) $t=5 T / 8, \alpha=22.83^{\circ}$

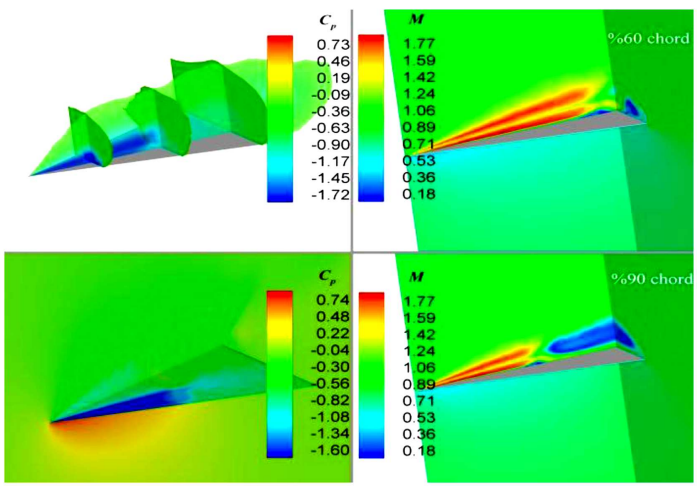

(g) $t=3 T / 4, \alpha=24^{\circ}$

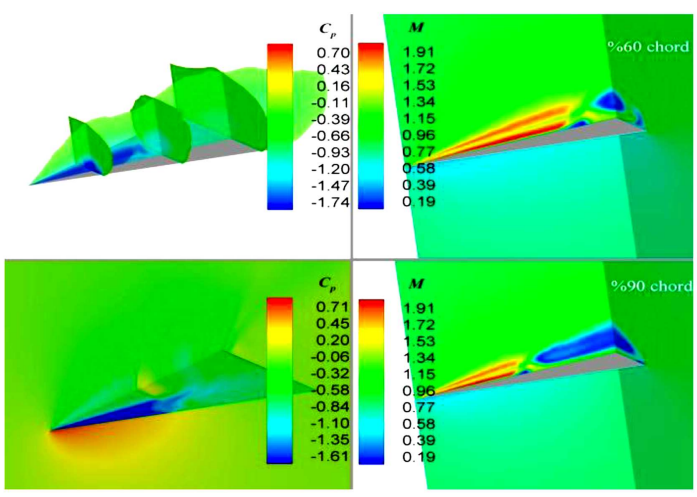

(h) $t=7 T / 8, \alpha=22.83^{\circ}$

Figure 8. Flow structure variations around the wing during a pitching cycle with $M_{\infty}=0.8, \alpha_{0}=20^{\circ}, \bar{\alpha}=4^{\circ}$, and $f=10 \mathrm{~Hz}$. 


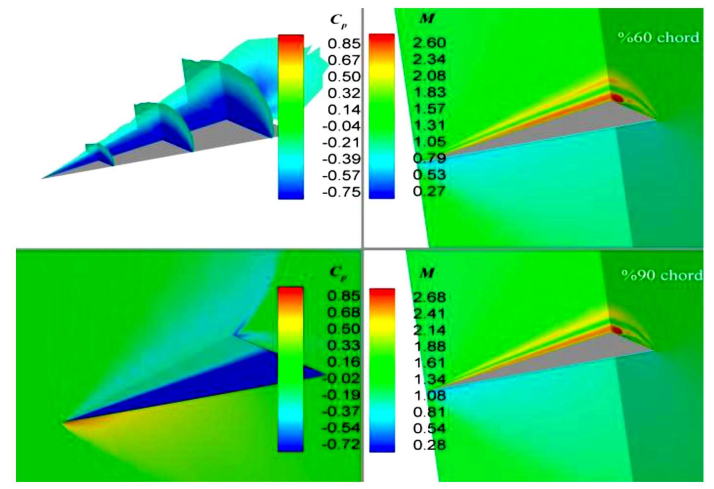

(a) $t=0, \alpha=20^{\circ}$

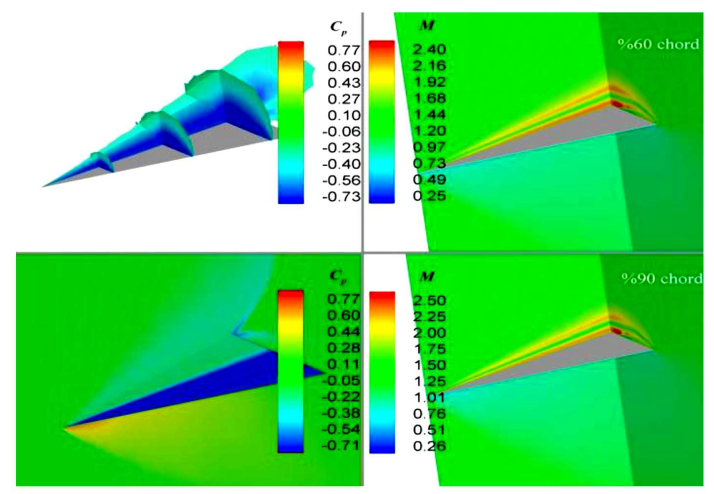

(b) $t=T / 8, \alpha=17.17^{\circ}$

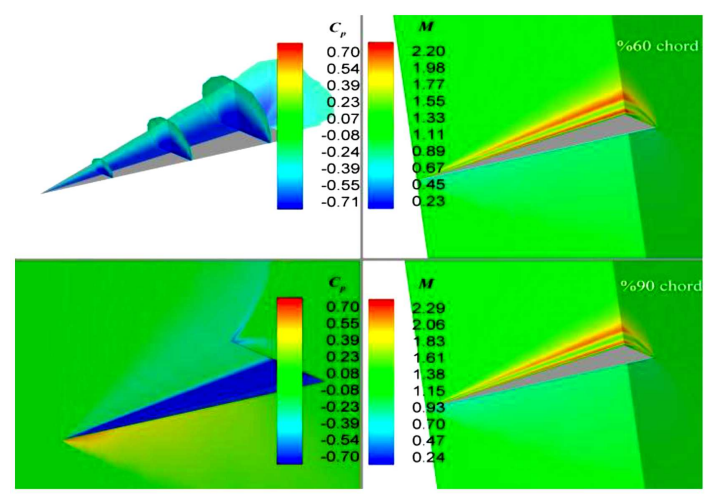

(c) $t=T / 4, \alpha=16^{\circ}$

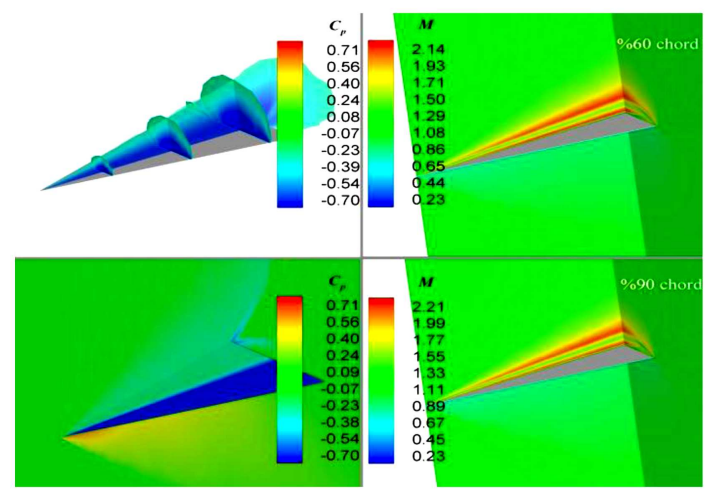

(d) $t=3 T / 4, \alpha=17.17^{\circ}$

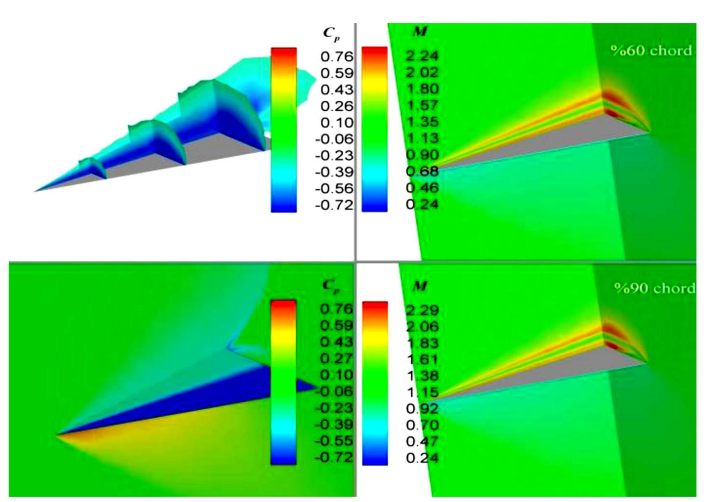

(e) $t=T / 2, \alpha=20^{\circ}$

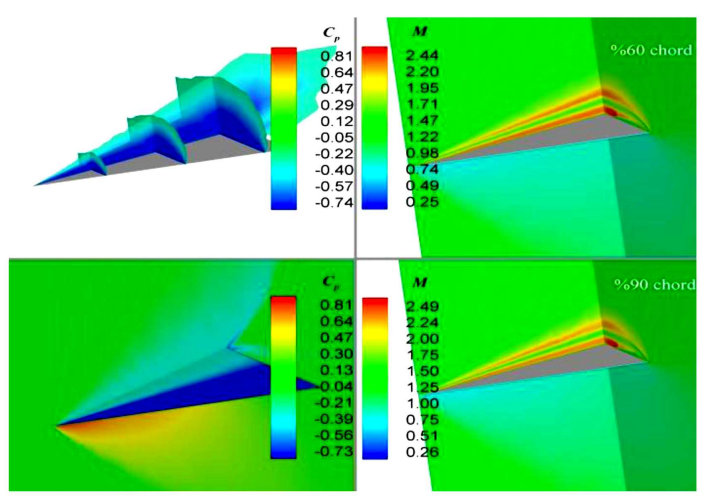

(f) $t=5 T / 8, \alpha=22.83^{\circ}$

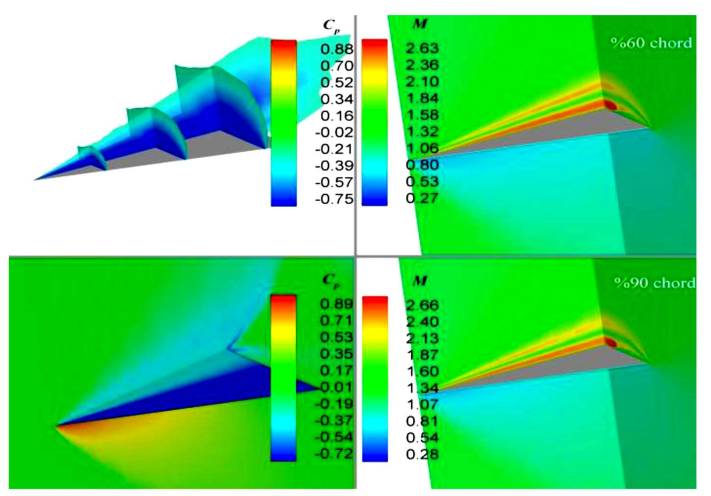

(g) $t=3 T / 4, \alpha=24^{\circ}$

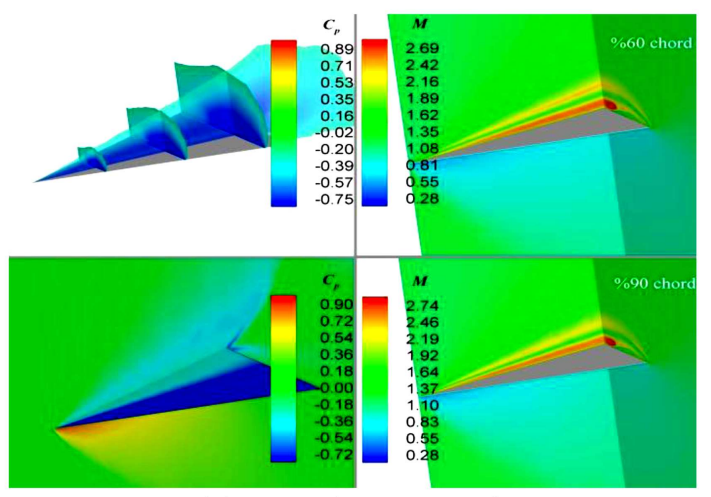

(h) $t=7 T / 8, \alpha=22.83^{\circ}$

Figure 9. Flow structure variations around the wing during a pitching cycle with $M_{\infty}=1.2, \alpha_{0}=20^{\circ}, \bar{\alpha}=4^{\circ}$, and $f=10 \mathrm{~Hz}$. 


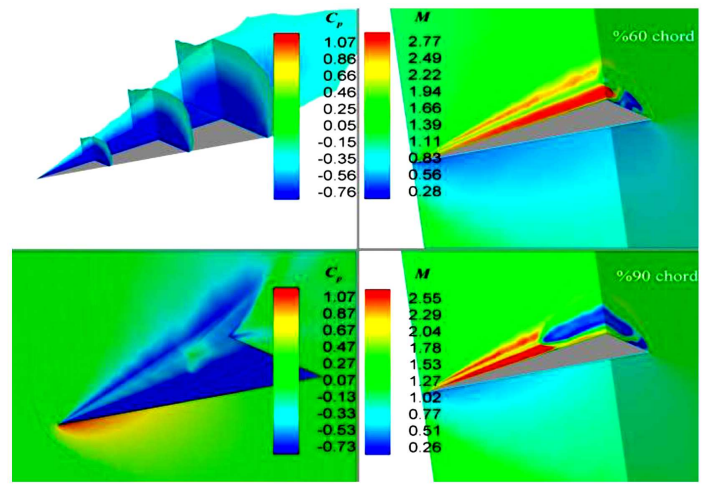

(a) $t=0, \alpha=30^{\circ}$

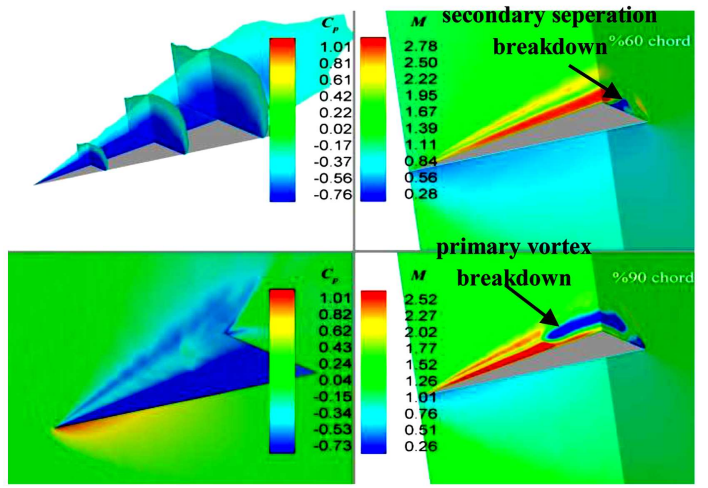

(b) $t=T / 8, \alpha=27.17^{\circ}$

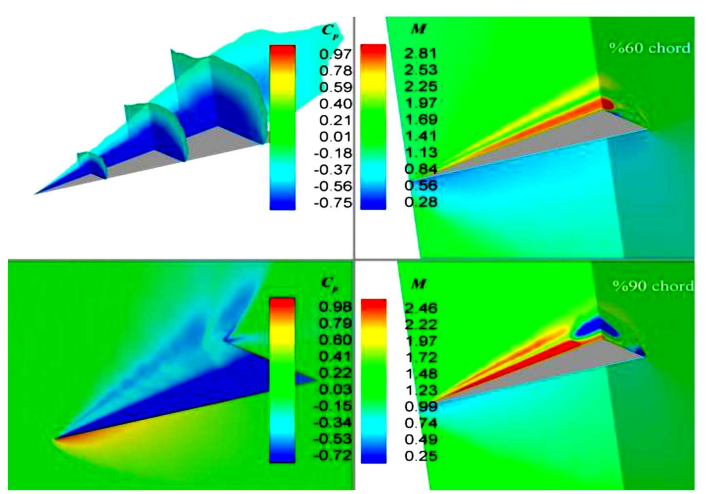

(c) $t=T / 4, \alpha=26^{\circ}$

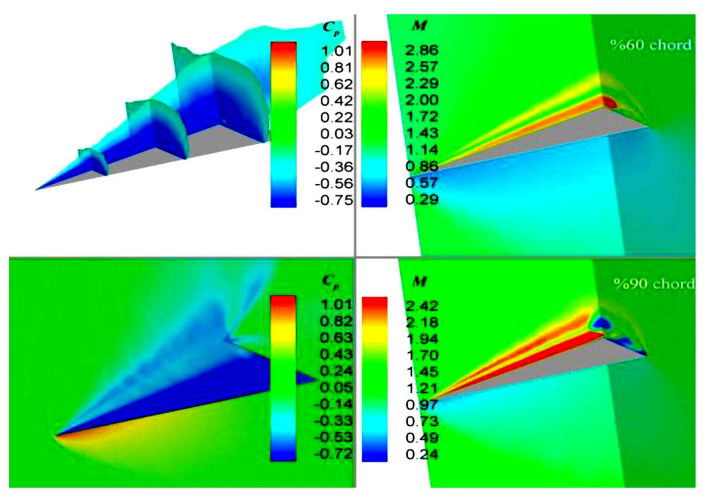

(d) $t=3 T / 4, \alpha=27.17^{\circ}$

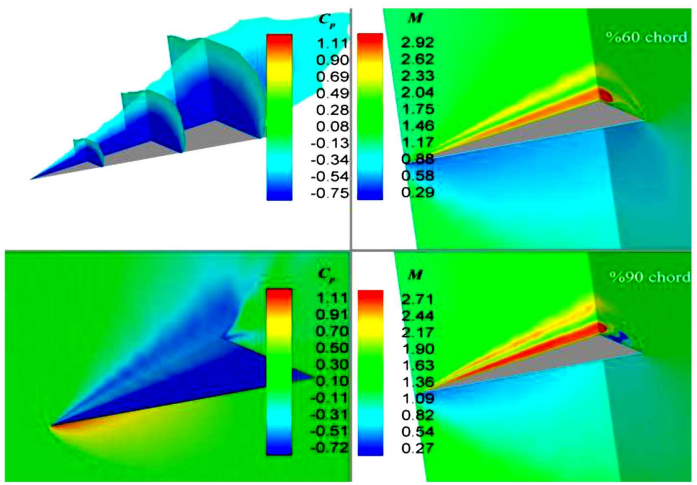

(e) $t=T / 2, \alpha=30^{\circ}$

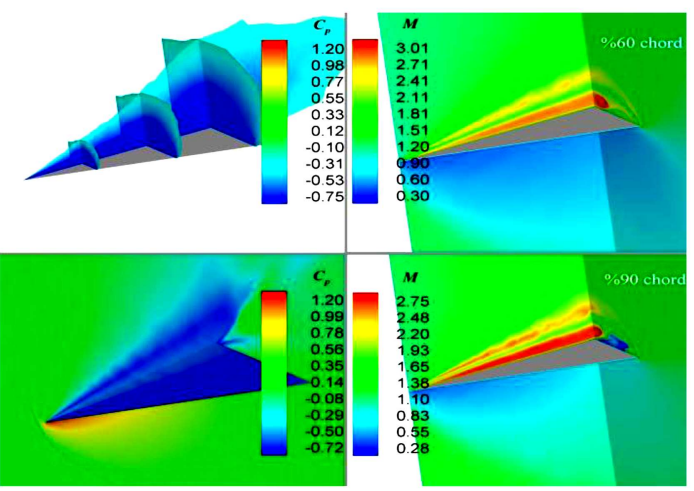

(f) $t=5 T / 8, \alpha=32.83^{\circ}$

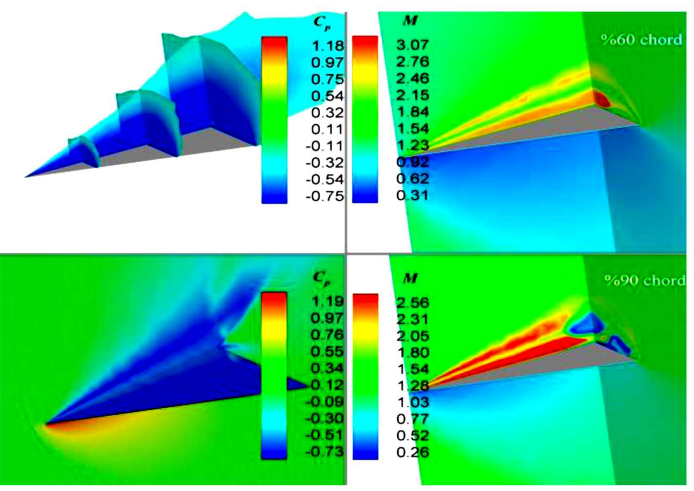

(g) $t=3 T / 4, \alpha=34^{\circ}$

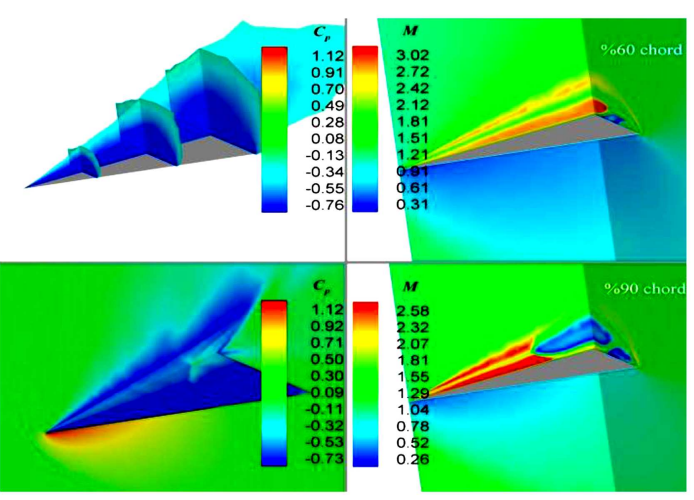

(h) $t=7 T / 8, \alpha=32.83^{\circ}$

Figure 10. Flow structure variations around the wing during a pitching cycle with $M_{\infty}=1.2, \alpha_{0}=30^{\circ}, \bar{\alpha}=4^{\circ}$, and $f=10 \mathrm{~Hz}$. 


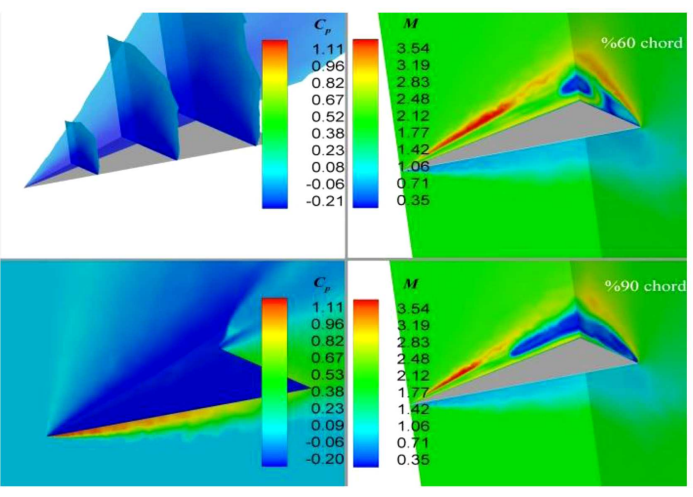

(a) $t=0, \alpha=40^{\circ}$

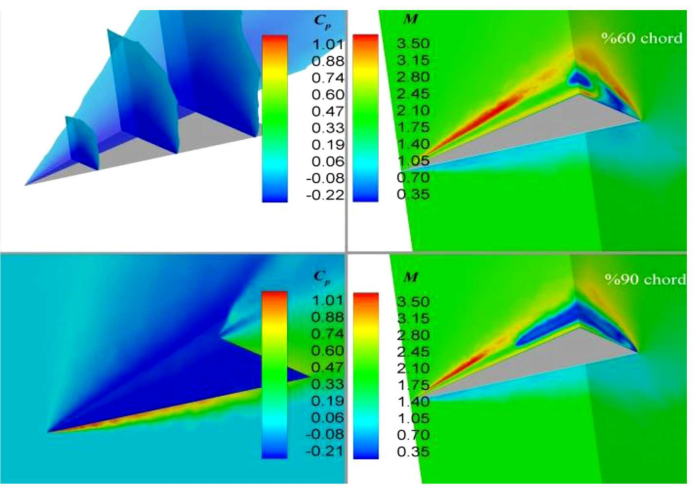

(b) $t=T / 8, \alpha=37.17^{\circ}$

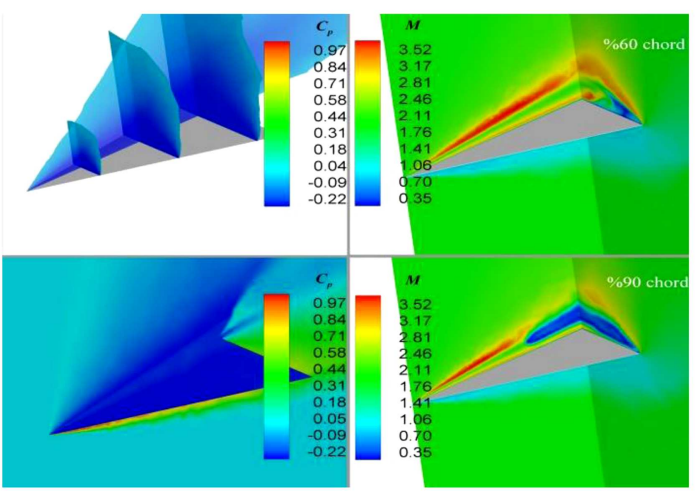

(c) $t=T / 4, \alpha=36^{\circ}$

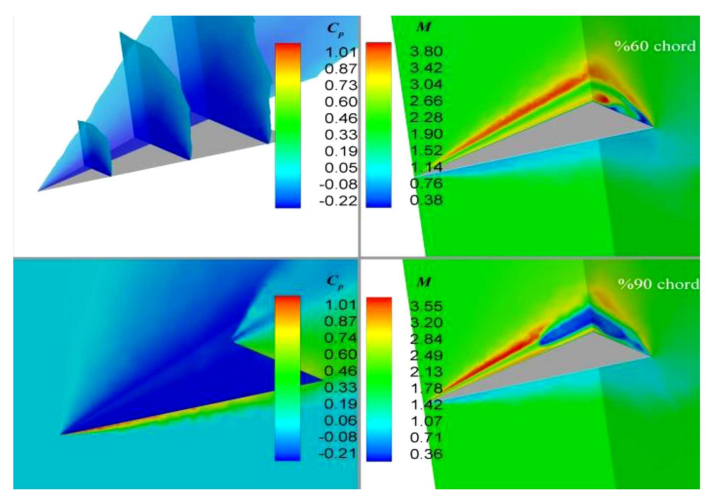

(d) $t=3 T / 4, \alpha=37.17^{\circ}$

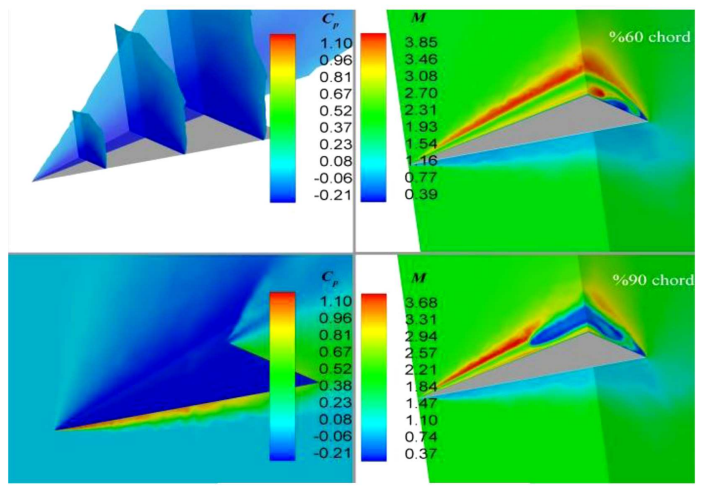

(e) $t=T / 2, \alpha=40^{\circ}$

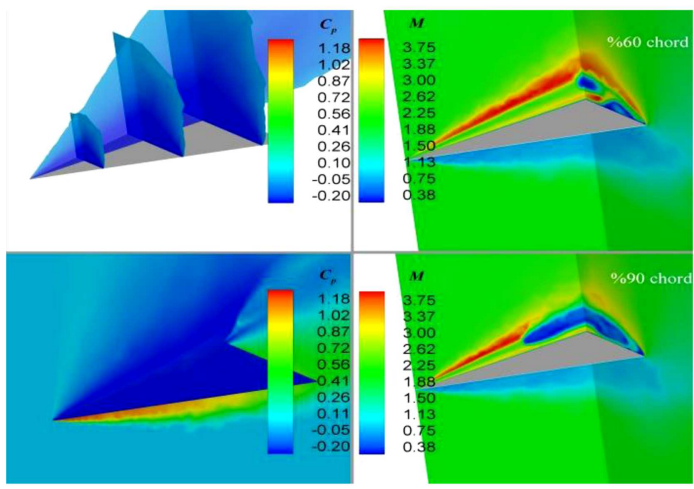

(f) $t=5 T / 8, \alpha=42.83^{\circ}$

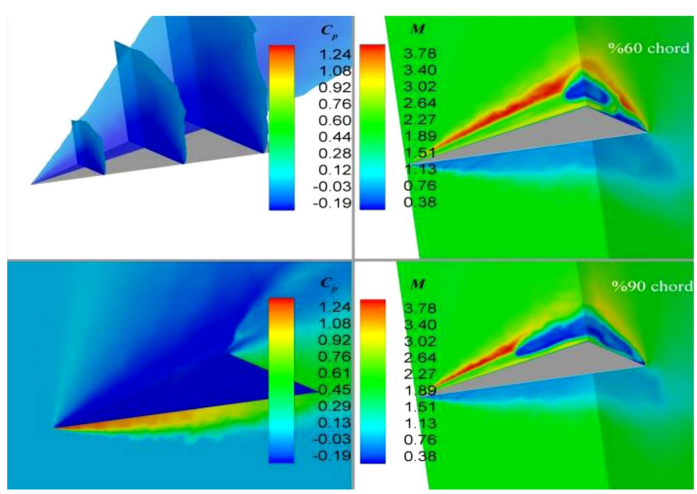

(g) $t=3 T / 4, \alpha=44^{\circ}$

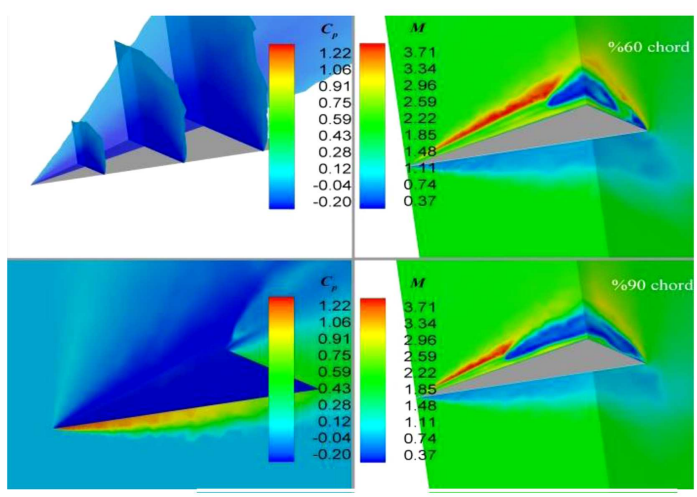

(h) $t=7 T / 8, \alpha=42.83^{\circ}$

Figure 11. Flow structure variations around the wing during a pitching cycle with $M_{\infty}=2, \alpha_{0}=40^{\circ}, \bar{\alpha}=4^{\circ}$, and $f=10 \mathrm{~Hz}$. 
after completing three cycles from starting the pitching motion.

As seen in Figure 8(a)-(h) for $M_{\infty}=0.8, \alpha_{0}=$ $20^{\circ}$, and $\bar{\alpha}=4^{\circ}$, onset of primary vortex breakdown occurs before flow reaches the $60 \%$ chordwise station. This breakdown is characterized by abrupt transition from a jetlike to a wake-like core of the vortex, accompanied by a substantial increase in turbulence activity. Variations of breakdown location can be observed in the figure. With increase in Mach number, adverse pressure gradient in the chordwise direction becomes smaller and the location of breakdown moves downstream. Therefore, it is seen in Figure 9(a)-(h) that for $M_{\infty}=1.2, \alpha_{0}=20^{\circ}$, and $\bar{\alpha}=4^{\circ}$, the vortex does not break down over the wing surface. However, Figure 10(a)-(h) shows that by increasing angle of attack, breakdown can occur in supersonic condition. For $M_{\infty}=1.2, \alpha_{0}=30^{\circ}$, and $\bar{\alpha}=4^{\circ}$, onset of primary vortex breakdown occurs before flow reaches the $90 \%$ chordwise station. Of course, it can be seen in the figure that secondary separation experiences breakdown before primary vortex. Figure 11(a)-(h) shows that for $M_{\infty}=2, \alpha_{0}=40^{\circ}$, and $\bar{\alpha}=4^{\circ}$, primary vortex breakdown occurs and its location oscillates around the $60 \%$ chordwise station. For simulated cases with $M_{\infty}=2$ and lower mean angles of attack, vortex breakdown does not happen. Figures 9 to 11 show that variation of pressure coefficient on the wing surface is very little in supersonic Mach numbers.

Figure 12 shows the effect of free-stream Mach number on vortex breakdown chordwise location hysteresis loop in subsonic regime. The loop direction is clockwise. As seen in the figure, shapes of the loops are similar, but breakdown location is further downstream in $M_{\infty}=0.8$ as it is expected. Breakdown does not occur at higher Mach numbers for this angle of attack.

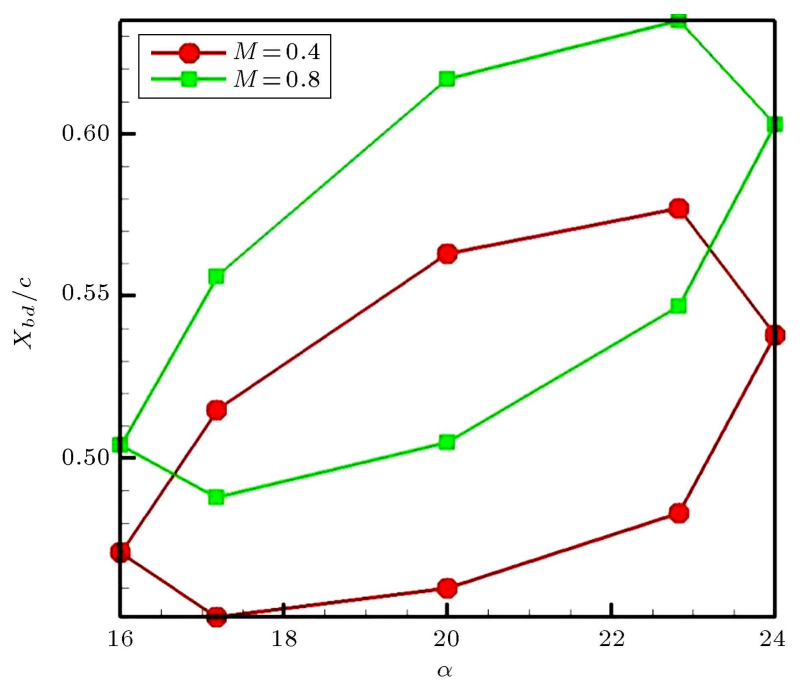

Figure 12. Mach number effect on vortex breakdown chordwise location at $\alpha_{0}=20^{\circ}, \bar{\alpha}=4^{\circ}$, and $f=10 \mathrm{~Hz}$.
Figures 13 and 14 show the vortex breakdown chordwise location hysteresis loop at $M_{\infty}=1.2, \bar{\alpha}=$ $30^{\circ}$, and pitch amplitudes of $4^{\circ}$ and $8^{\circ}$, respectively, with different frequencies. It is seen that increasing frequency makes the loop slightly narrower, i.e. the amplitude of breakdown location oscillation becomes more limited. Also, it is seen that at amplitude of $4^{\circ}$, changing the frequency from $10 \mathrm{~Hz}$ to $50 \mathrm{~Hz}$ shifts the breakdown mean location downstream. If the wing were stationary, the quantity $X_{b d} / c$ would be maximum at minimum angle of attack and minimum at maximum angle of attack. However, it can be seen in the last three figures that this is not true for the oscillating wing and breakdown location has a large phase difference with wing motion. A time

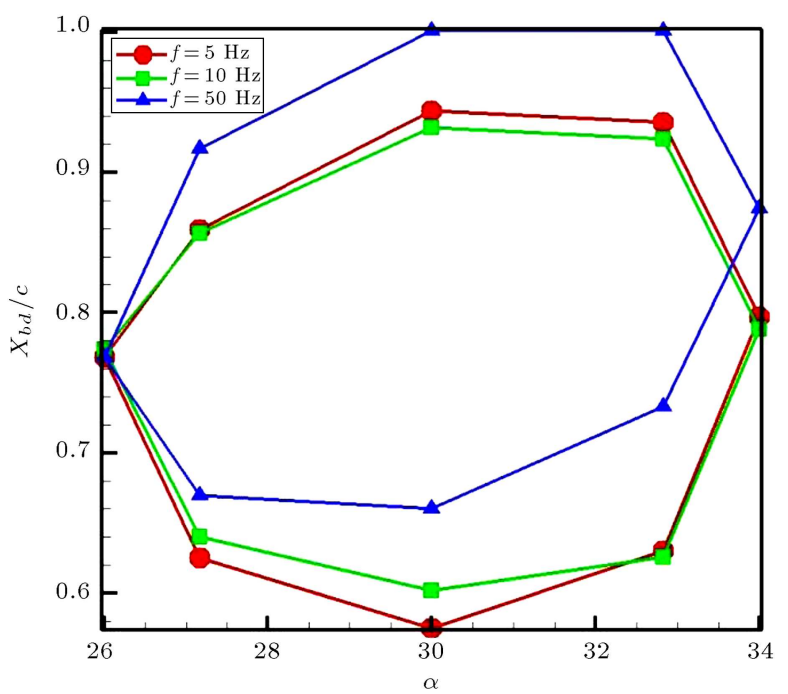

Figure 13. Frequency effect on vortex breakdown chordwise location at $M_{\infty}=1.2, \alpha_{0}=30^{\circ}$, and $\bar{\alpha}=4^{\circ}$.

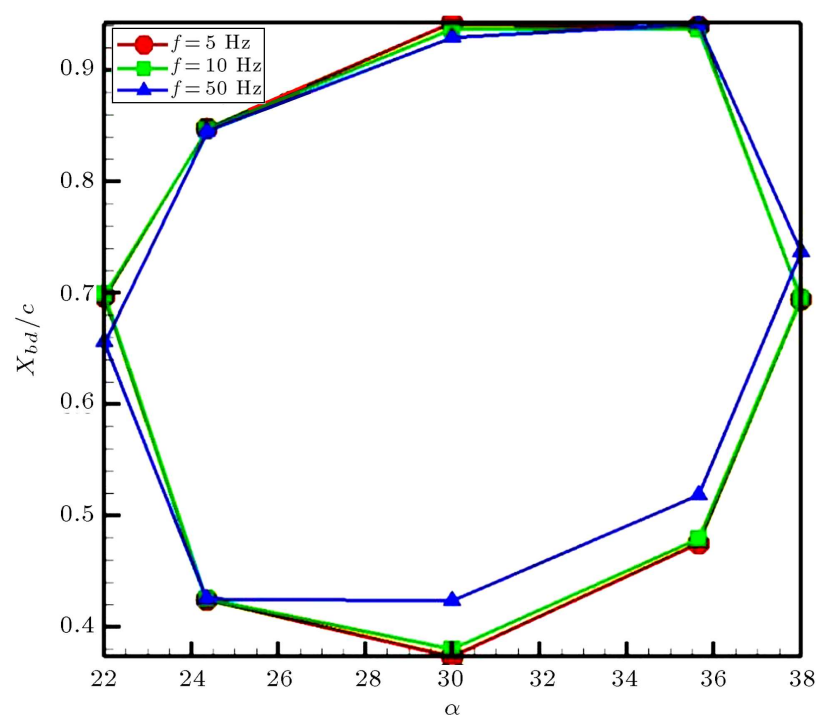

Figure 14. Frequency effect on vortex breakdown chordwise location at $M_{\infty}=1.2, \alpha_{0}=30^{\circ}$, and $\bar{\alpha}=8^{\circ}$. 
delay also exists in flow pattern in pitching motion with respect to the stationary flow field. The authors showed that the time lag associated with flow pattern (vortex formation) was approximately $T / 8$. However, these two events have very different time scales. The time lag of flow pattern is very small compared to the large time lag of breakdown location.

\subsection{Aerodynamic coefficients}

Figures 15 to 23 show the lift or drag coefficients versus angle of attack hysteresis loops for different flow conditions. Directions of the loops are clockwise. In Figure 15 are compared the hysteresis loops for four different Mach numbers when other parameters are fixed. Fluctuations seen in the $M_{\infty}=0.4$ curve are

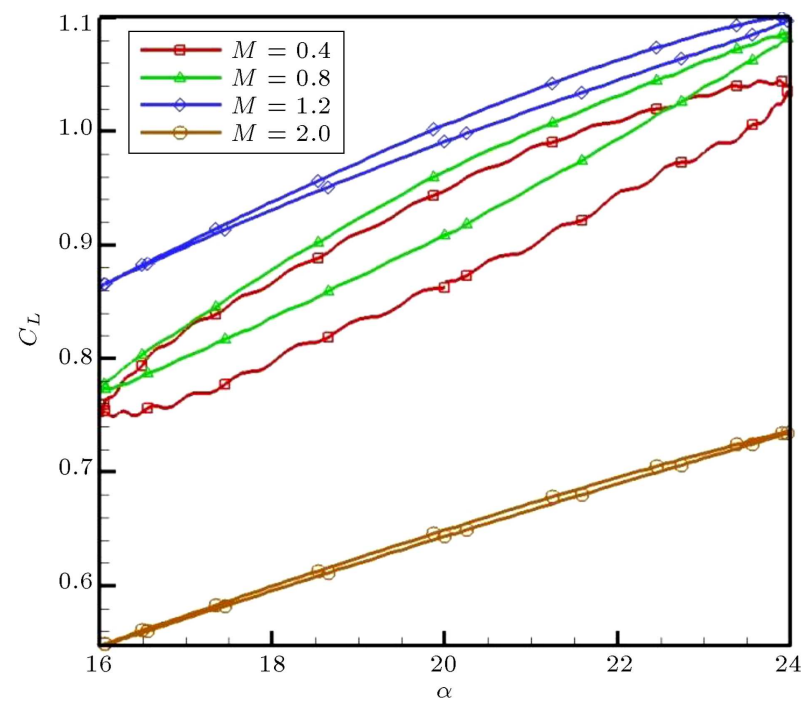

Figure 15. Frequency effect on lift coefficient at $M_{\infty}=1.2, \alpha_{0}=8^{\circ}$, and $\bar{\alpha}=4^{\circ}$.

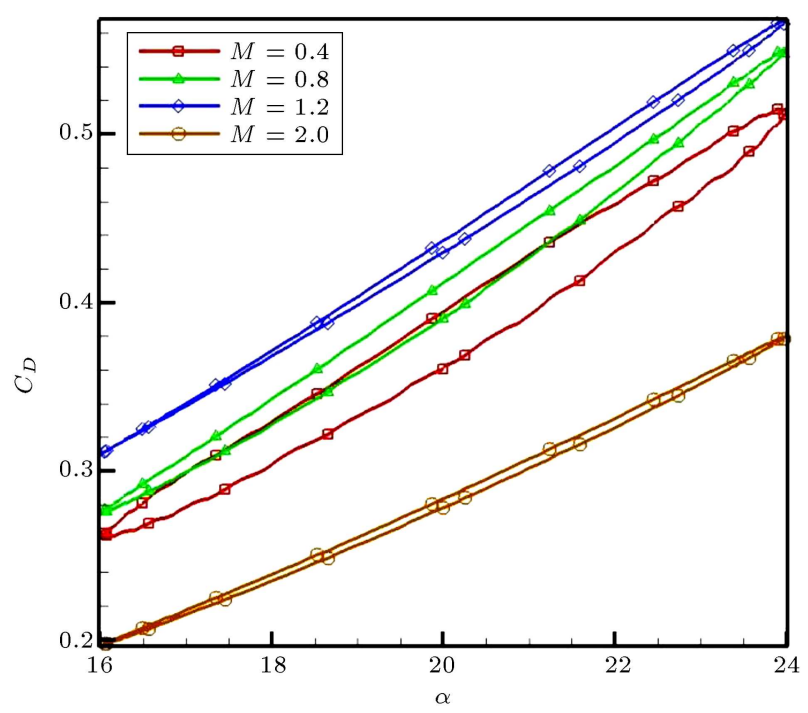

Figure 16. Mach number effect on lift coefficient at $\alpha_{0}=20^{\circ}, \bar{\alpha}=4^{\circ}$, and $f=10 \mathrm{~Hz}$.

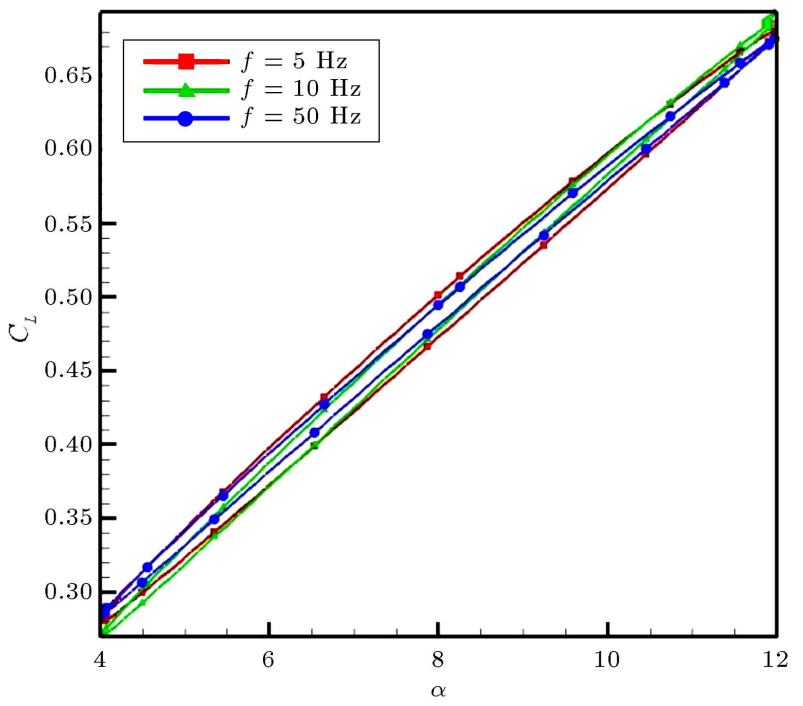

Figure 17. Mach number effect on drag coefficient at $\alpha_{0}=20^{\circ}, \bar{\alpha}=4^{\circ}$, and $f=10 \mathrm{~Hz}$.

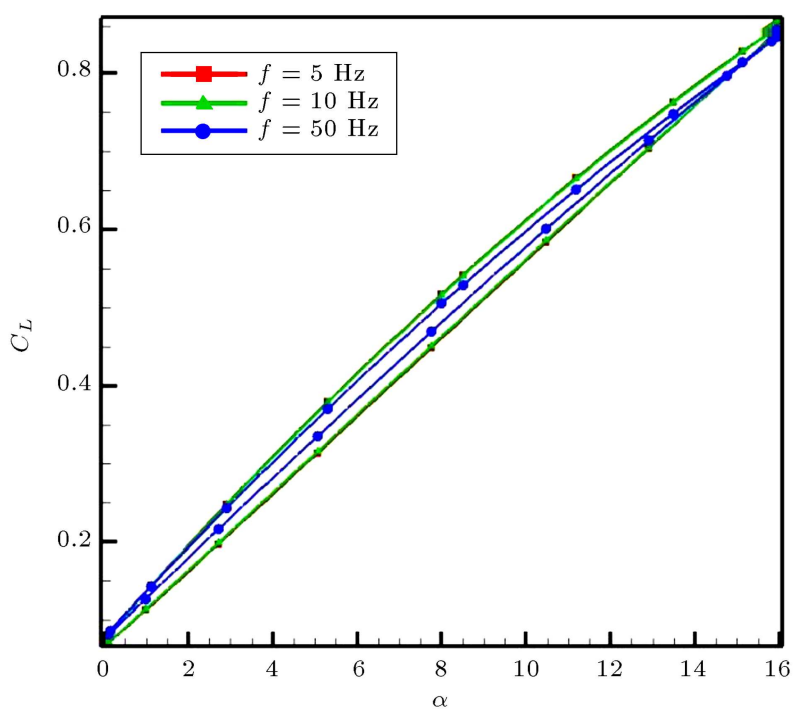

Figure 18. Frequency effect on lift coefficient at $M_{\infty}=1.2, \alpha_{0}=8^{\circ}$, and $\bar{\alpha}=8^{\circ}$.

due to inherent flow unsteadiness caused by vortex breakdown. They also exist in $M_{\infty}=0.8$ curve, though they are less obvious. Increasing Mach number increases the lift in subsonic cases and decreases it in supersonic cases. This trend is expected, as linear theory predicts it. It makes the loop narrower and decreases the curve slope at supersonic cases. A similar trend is observed for drag coefficient in Figure 16, although the hysteresis is small in drag coefficient compared to that in the lift coefficient.

Comparison of Figures 17 and 18 shows that the hysteresis loop is wider for lower frequencies at $\alpha_{0}=8^{\circ}$ and $\bar{\alpha}=4^{\circ}$, but it is vice versa at higher pitching amplitude of $\bar{\alpha}=8^{\circ}$. Noticing Figures 19 to 22, we find out that increasing mean angle of attack increases 


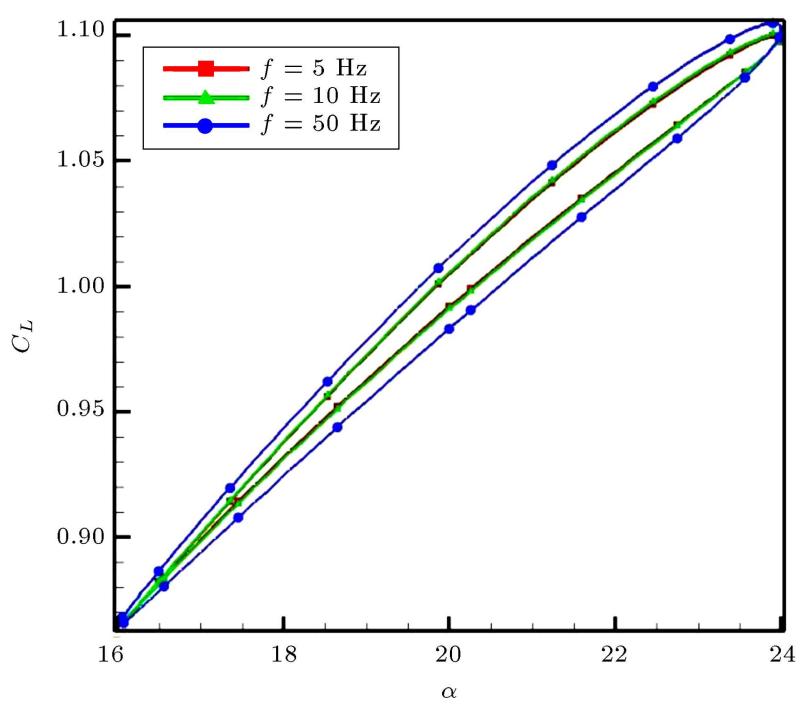

Figure 19. Frequency effect on lift coefficient at $M_{\infty}=1.2, \alpha_{0}=20^{\circ}$, and $\bar{\alpha}=4^{\circ}$.

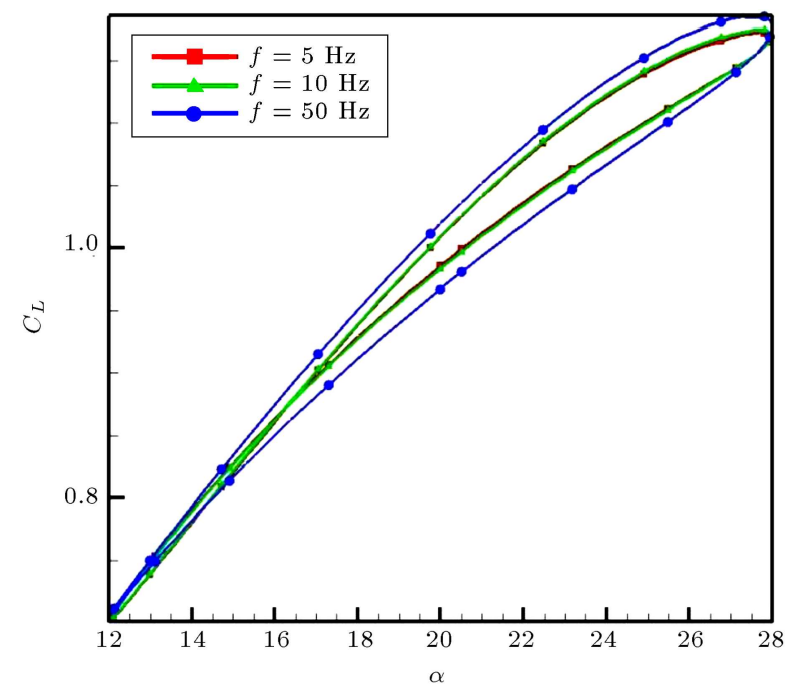

Figure 20. Frequency effect on lift coefficient at $M_{\infty}=1.2, \alpha_{0}=20^{\circ}$, and $\bar{\alpha}=8^{\circ}$.

the lift coefficient and widens the loop severely in cases where vortex breakdown exists. Also, it can be seen obviously that increasing oscillation amplitude changes the loop shape, especially at higher instantaneous angles of attack.

Figure 24 shows hysteresis loops associated with pitching moment coefficient about the mid-chord (axis of rotation) for four different Mach numbers when other parameters are fixed. Similar to lift and drag coefficients, the mean value of pitching moment coefficient increases in subsonic region and decreases in supersonic region. The loops have an approximately constant slope for $M_{\infty} \geq 0.8$. The amount of hysteresis is reduced with increasing Mach number, which results in narrower loops and less phase difference. Eight-shape phenomenon is observed for $M_{\infty}=0.8$.

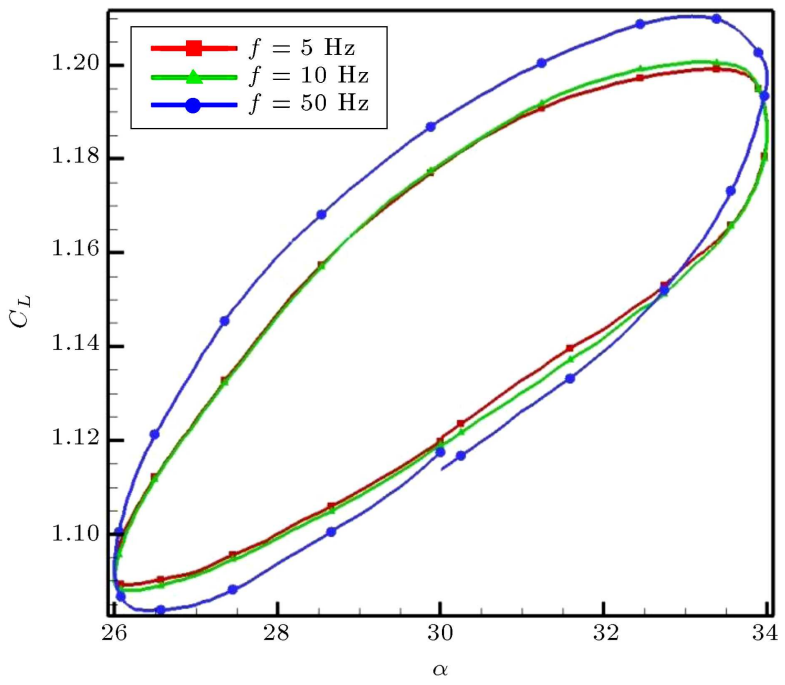

Figure 21. Frequency effect on lift coefficient at $M_{\infty}=1.2, \alpha_{0}=30^{\circ}$, and $\bar{\alpha}=4^{\circ}$.

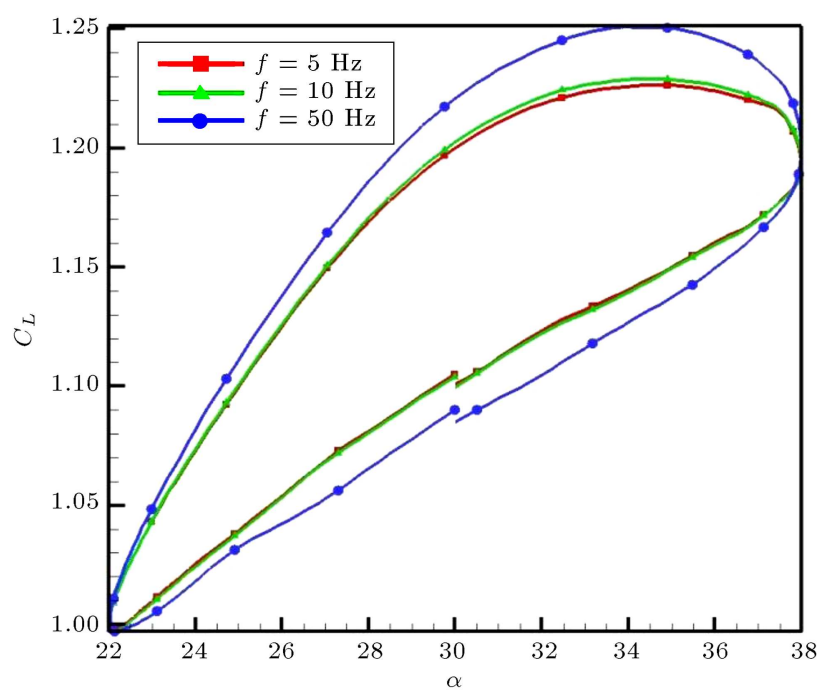

Figure 22. Frequency effect on lift coefficient at $M_{\infty}=1.2, \alpha_{0}=30^{\circ}$, and $\bar{\alpha}=8^{\circ}$.

\section{Conclusion}

Flow fields over a $60^{\circ}$ pitching delta wing with sharp leading edge, from subsonic to supersonic flow regimes, were computationally simulated using compressible 3D Navier-Stokes equations on an unstructured grid and a $k-\omega \mathrm{SST}$ solver. Local Mach number and pressure coefficient contours showed that vortex breakdown occurred for large enough angles of attack and the time delay associated with it was shown to be large compared to the flow structure variation time delay. For $M_{\infty}=0.8, \alpha_{0}=20^{\circ}$, and $\bar{\alpha}=4^{\circ}$, onset of primary vortex breakdown occurred before flow reached the $60 \%$ chordwise station. For $M_{\infty}=1.2, \alpha_{0}=20^{\circ}$, and $\bar{\alpha}=4^{\circ}$, the vortex did not break down over the wing surface. For $M_{\infty}=1.2, \alpha_{0}=30^{\circ}$, and $\bar{\alpha}=4^{\circ}$, 


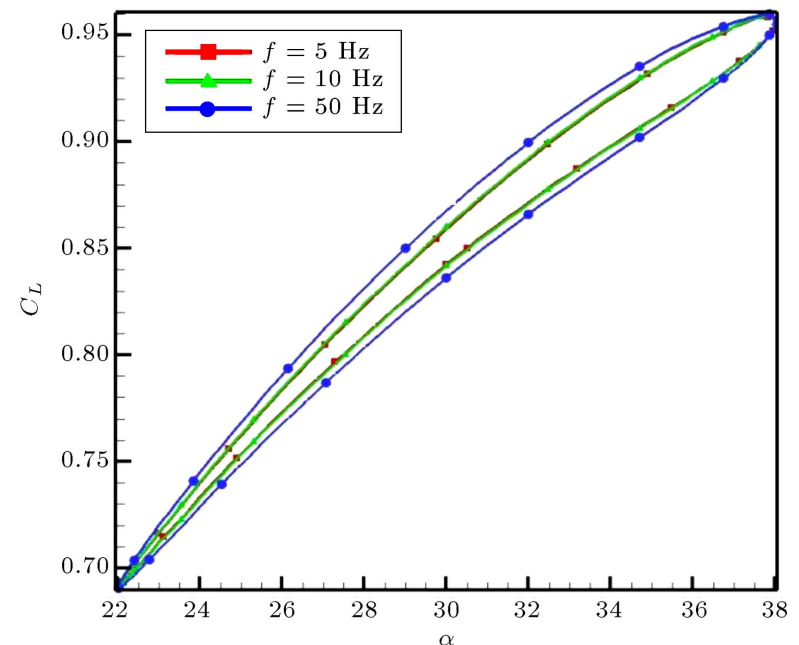

Figure 23. Frequency effect on lift coefficient at $M_{\infty}=2$, $\alpha_{0}=30^{\circ}$, and $\bar{\alpha}=8^{\circ}$.

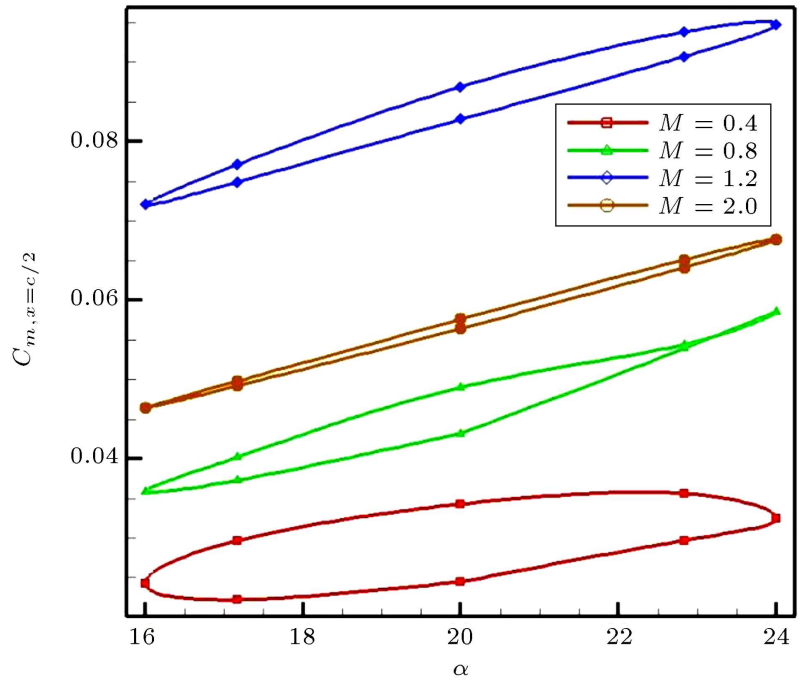

Figure 24. Mach number effect on pitching moment coefficient around mid-chord at $\alpha_{0}=20^{\circ}, \bar{\alpha}=4^{\circ}$, and $f=10 \mathrm{~Hz}$.

onset of primary vortex breakdown occurred before flow reached the $90 \%$ chordwise station, but secondary separation experienced breakdown before primary vortex. For $M_{\infty}=2, \alpha_{0}=40^{\circ}$, and $\bar{\alpha}=4^{\circ}$, primary vortex breakdown occurred and its location oscillated around the $60 \%$ chordwise station. The effects of flow parameters, namely, Mach number, mean angle of attack, motion amplitude, and frequency, on the hysteresis loops of vortex breakdown location over the wing and aerodynamic coefficients were investigated. Increasing free-stream Mach number delayed the vortex breakdown. Increasing frequency made the vortex breakdown location loop slightly narrower, i.e. the amplitude of breakdown location oscillation became more limited. Increasing Mach number made the aerodynamic coefficient loops narrower and decreased the curve slope at supersonic cases. Increasing mean angle of attack increased the lift coefficient and widened the loop in cases where vortex breakdown existed. Increasing oscillation amplitude changed the loop shape, especially at higher instantaneous angles of attack.

\section{Nomenclature}

$\begin{array}{ll}c & \text { Chord } \\ C_{L} & \text { Lift coefficient } \\ C_{D} & \text { Drag coefficient } \\ C_{p} & \text { Pressure coefficient } \\ f & \text { Frequency } \\ k & \text { Reduced frequency } \\ M & \text { Mach number } \\ t & \text { Time } \\ T & \text { Period } \\ U & \text { Velocity }\end{array}$

$X \quad$ Chordwise coordinate from the apex of the wing

$a$

$\bar{\alpha}$

$\Lambda$

Angle of attack

Amplitude

Sweep angle

$\omega=2 \pi f \quad$ Angular velocity

\section{Subscripts}

$\begin{array}{ll}b d & \text { Breakdown } \\ N & \text { Component normal to the leading edge } \\ 0 & \text { Mean } \\ \infty & \text { Free-stream }\end{array}$

\section{References}

1. Gursul, I., Gordnier, R. and Visbal, M. "Unsteady aerodynamics of nonslender delta wings", Progress in Aerospace Sciences, 41(7), pp. 515-557 (2005).

2. Gursul, I. "Recent developments in delta wing aerodynamics", The Aeronautical Journal, 108, pp. 437-452 (2004).

3. Rockwell, D. "Three-dimensional flow structure on delta wings at high angle-of-attack: experimental concepts issues", 31st Aerospace Science Meeting and Exhibit, Reno, Nevada, USA, January 11-14, paper no. 93-0550 (1993).

4. Visbal, M.R. "Computational and physical aspects of vortex breakdown on delta wings", 33rd Aerospace Science Meeting and Exhibit, Reno, Nevada, USA, January 9-12, paper no. 95-0585 (1993).

5. Atta, R. and Rockwell, D. "Hysteresis of vortex development and breakdown on an oscillating delta wing", AIA A Journal, 25(11), pp. 1512-1513 (1987). 
6. LeMay, S.P., Batill, S.M. and Nelson, R.C. "Vortex dynamics on a pitching delta wing", Journal of Aircraft, $\mathbf{2 7}(2)$, pp. 131-138 (1990).

7. Gursul, I. and Yang, H. "On fluctuations of vortex breakdown location", Physics of Fluids, 7(1), pp. 229231 (1995).

8. Gursul, I. and Ho, C.M. "Vortex breakdown over delta wings in unsteady freestream", AIA A Journal, 32(2), pp. 433-436 (1994).

9. Lin, J.C. and Rockwell, D. "Transient structure of vortex breakdown on a delta wing", AIAA Journal, 33(1), pp. 6-12 (1995).

10. Jones, M., Hashimoto, A. and Nakamura, Y. "Criteria for vortex breakdown above high-sweep delta wings", AIA A Journal, 47(10), pp. 2306-2320 (2009).

11. Goruney, T. and Rockwell, D. "Effect of pitch rate on near-surface topology on a delta wing", AIA A Journal, 48(6), pp. 1207-1220 (2010).

12. Jian, L., Haisheng, S., Zhitao, L. and Zhixiang, X. "Numerical investigation of unsteady vortex breakdown past $80^{\circ} / 65^{\circ}$ double-delta wing", Chinese Journal of Aeronautics, 27(3), pp. 521-530 (2014).

13. Stanbrook, A. and Squire, L.C. "Possible types of flow at swept leading edges", Aeronautical Quarterly, 15(2), pp. 72-78 (1964).

14. Miller, D.S. and Wood, R.M. "Leeside flows over delta wings at supersonic speeds", Journal of Aircraft, 21(9), pp. 680-686 (1984).

15. Szodruch, J.G. and Peake, D.J. "Leeward flow over delta wings at supersonic speeds", Report NASA-TM No. 81187 (1980).

16. Seshadri, S.N. and Narayan, K.Y. "Possible types of flow on lee-surface of delta wings at supersonic speeds", The Aeronautical Journal, 5, pp. 185-199 (1988).

17. Brodetsky, M.D., Krause, E., Nikiforov, S.B., Pavlov, A.A., Kharitonov, A.M. and Shevchenco, A.M. "Evolution of vortex structures on leeward side of a delta wing", Journal of Applied Mechanics and Technical Physics, 42(2), pp. 243-254 (2001).

18. Brodetsky, M.D. and Shevchenco, A.M. "Some features of a separated flow and supersonic vortex structure at the leeside of a delta wing", Proc. of the IUTAM Symp. on Separated Flows and Jets, Berlin-Heidelberg, Germany, 9-13 July, pp. 341-344 (1990).

19. Imai, G., Fujii, K. and Oyama, A. "Computational analyses of supersonic flows over a delta wing at high angles of attack", 25th International Congress of the Aeronautical Sciences (ICAS), Hamburg, Germany, 38 September (2006).

20. Oyama, A., Ito, M., Imai, G., Tsutsumi, S., Amitani, N. and Fujii, K. "Mach number effect on flow field over a delta wing in supersonic region", 46th AIAA Aerospace Sciences Meeting and Exhibit, Reno, Nevada, USA, 7-10 January, paper no. 354 (2008).

21. Schiavetta, L.A., Boelens, O.J. and Fritz, W. "Analysis of transonic flow on a slender delta wing using CFD", 24th Applied Aerodynamics Conf., San Francisco, California, USA, 5-8 June, paper no. 3171 (2006).

22. Schiavetta, L.A., Badcock, K.J. and Cummings, R.M. "Comparison of DES and URANS for unsteady vortical flows over delta wings", 46th AIAA Aerospace Sciences Meeting and Exhibit, Reno, Nevada, USA, 811 January, paper no. 1085 (2007).

23. Younis, Y., Bibi, A., Haque. A.U., and Khushnood, S. "Vortical flow topology on windward and leeward side of delta wing at supersonic speed", Journal of Applied Fluid Mechanics, 2(2), pp. 13-21 (2001).

24. Hadidoolabi, M. and Ansarian, H. "Computational investigation of the flow structure over a pitching delta wing at supersonic speeds", Proceedings of the Institution of Mechanical Engineers, Part G: Journal of Aerospace Engineering, 230(7), pp. 1334-1347 (2016). DOI: $10.1177 / 0954410015608207$.

25. Gordnier, R.E. and Visbal, M.R. "High-order simulations of low sweep delta wing flows using ILES and hybrid RANS/ILES models", 44th AIAA Aerospace Sciences Meeting and Exhibit, Reno, Nevada, USA, 912 January, paper no. 504 (2006).

\section{Biographies}

Mostafa Hadidoolabi is an Associate Professor and PhD advisor at Malek Ashtar University of Technology, Iran. His area of research includes CFD, applied aerodynamics, wind tunnel design, etc.

Hossein Ansarian received his BS and MS degrees from Sharif University of Technology in 2007 and 2009, respectively, and his $\mathrm{PhD}$ degree from Malek Ashtar University of Technology in 2016. Currently, His main research interest is the application of CFD in simulation of unsteady aerodynamic flows, including delta wing flows. 